\title{
Numerical Simulation of Temperature Distribution in the Glass Fiber Epoxy Composites during Microwave Curing
}

\author{
X Zhang, X L Chang, R L Ma, L Zhang, X D Chen and Q Zhang \\ Department of Power Engineering, Xi'an Hi-Tech Institute, Xi'an, 710025, China
}

\begin{abstract}
A three-dimensional coupled model of electromagnetic field, temperature field and curing degree field was established. Based on this model, the simulation of microwave curing process of glass fiber epoxy ring was realized, and the temperature distribution at different time was obtained. Numerical results indicate that the temperature difference within the composite ring is mainly formed in the initial stage during microwave curing.
\end{abstract}

\section{Introduction}

Advanced polymer matrix composites have the advantages of high strength-to-weight and stiffness-to-weight ratios, as well as good corrosion resistance and fatigue resistance performance, which have been widely used in aerospace and aircraft $[1,2]$. Currently, the main curing technique for fabricating polymer composites is conventional thermal curing technique, which has some disadvantages such as high operational costs and long processing cycle3. Microwave curing technique, however, has been shown to be a promising alternative to solve the existent problems with some superiorities including fast heating rate, selective and volumetric heating, energy saving, easy controllability and high curing efficiency 4.

During microwave curing process, the electromagnetic field and temperature distribution in different positions of the composites at different time are difficult to obtain. In addition, the conventional method by experiments will consume a lot of manpower, material and financial resources. In view of these constraints, it is very important to carry out the numerical simulation of microwave curing process. The realization of numerical simulations on microwave curing processes of epoxy composites will provide a feasible method for the study of microwave curing technique.

In this paper, the modeling and numerical simulations of multiphysics coupling on microwave curing processes of epoxy composites are carried out based on electromagnetic, heat transfer and cure reaction kinetics theories, a three-dimensional coupled model of electromagnetic field, temperature field and curing degree field is established through analysis of microwave curing process of glass fiber epoxy composites, and the model includes two internal heat sources.

\section{Numerical modeling}

The microwave curing process of epoxy matrix composites is a nonlinear multiphysics coupling process involving two internal heat sources. In order to establish the model, the electromagnetic field, temperature field and curing degree field need to be simulated at the same time. The Maxwell equations, heat conduction equation and curing kinetics equation are combined to obtain the temperature distribution in the composites.

\subsection{Maxwell equations}

The theoretical basis for solving the electromagnetic field distribution in the composite microwave curing process is Maxwell equations

$$
\begin{aligned}
& \nabla \times \mathrm{E}=-\partial \mathrm{B} / \partial t \\
& \nabla \times \mathrm{H}=\mathrm{J}+\partial \mathrm{D} / \partial t \\
& \nabla \times \mathrm{D}=\rho \\
& \nabla \times \mathrm{B}=0
\end{aligned}
$$

Where $E, H, D, B, J, \rho$ respectively represent the electric field strength, magnetic field strength, electric flux density, magnetic flux density, current density and charge density. According to the formulas (1) to (4), together with appropriate boundary conditions, the distribution of the electromagnetic field inside the microwave oven and inside the composites can be solved.

\subsection{Heat conduction equation}

According to the law of conservation of energy, the three-dimensional transient heat conduction equation of the composites during microwave curing is given as 


$$
\frac{\partial}{\partial x}\left(K_{x} \frac{\partial T}{\partial x}\right)+\frac{\partial}{\partial y}\left(K_{y} \frac{\partial T}{\partial y}\right)+\frac{\partial}{\partial z}\left(K_{z} \frac{\partial T}{\partial z}\right)+P+Q_{V}=\rho_{c} C_{p c} \frac{\partial T}{\partial t}
$$

Where $P$ and $Q v$ are the two internal heat sources in the composites microwave curing process. Among them, $P$ is the microwave energy absorbed by the composites per unit volume at a certain moment. The microwave energy absorbed by the composites can be calculated according to the Poynting theorem[6]:

$$
P=2 \pi f \varepsilon \varepsilon^{\prime \prime} E^{2}
$$

Where $P$ is the microwave energy absorbed by the composites $\left(\mathrm{W} / \mathrm{m}^{3}\right)$; $\mathrm{f}$ is the microwave frequency, the value is $2.45 \mathrm{GHz}$; $\varepsilon$ is the free space permittivity, the value is $8.854 \times 10^{-12} \mathrm{~F} / \mathrm{m} ; \varepsilon^{\prime \prime} \quad$ is the dielectric loss factor of the material; $E$ is the electric field strength $(\mathrm{V} / \mathrm{m})$. In the numerical simulation of composites microwave curing process, the electric field strength $E$ is obtained by solving Maxwell equations.

$Q v$ is the reaction heat released by the curing reaction of the resin matrix, which is expressed as follows

$$
Q_{V}=\rho_{r} V_{r} H_{r} d \alpha / d t
$$

Substituting (6) and (7) into (5), the three-dimensional transient heat conduction equation for the microwave curing process can be expressed as

$$
\rho_{c} C_{p c} \frac{\partial T}{\partial t}=\frac{\partial}{\partial x}\left(K_{x} \frac{\partial T}{\partial x}\right)+\frac{\partial}{\partial y}\left(K_{y} \frac{\partial T}{\partial y}\right)+\frac{\partial}{\partial z}\left(K_{z} \frac{\partial T}{\partial z}\right)+2 \pi f \varepsilon \varepsilon^{\prime \prime} E^{2}+\rho_{r} V_{r} H_{r} \frac{d \alpha}{d t}
$$

\subsection{Curing kinetics equation}

The phenomenological curing reaction kinetics model as follows

$$
\begin{aligned}
& d \alpha / d t=k(T)(1-\alpha)^{n} \\
& k(T)=A \times e^{(-\Delta E / R T)}
\end{aligned}
$$

Where $\alpha$ is the curing degree, $\mathrm{n}$ is the reaction order, $\mathrm{k}$ is the reaction rate constant, $\mathrm{A}$ is the frequency factor, and $\Delta E$ is the activation energy of the resin curing reaction.

\section{4. boundary condition}

The sample will conduct convective heat transfer with air, and the boundary equation can be expressed as

$$
-k\left(\frac{\partial T}{\partial n}\right)_{\mathrm{W}}=h\left(T_{\mathrm{W}}-T_{a}\right)
$$

Where $\mathrm{k}$ is coefficient of heat conduction, $n$ is Surface normal direction, $T_{\mathrm{W}}$ is The temperature of the boundary, $T_{a}$ is ambient temperature, $h$ is Convective heat transfer coefficient.

\section{Simulation Process}

The cavity of the microwave curing oven used in this study is hemispherical with a rated of power $700 \mathrm{~W}$ and a operating frequency of $2.45 \mathrm{GHz} 7$. The same glass fiber/epoxy composite ring is selected as in Ref8, whose thickness, width and inner diameter are $3 \mathrm{~mm}, 6 \mathrm{~mm}$ and $150 \mathrm{~mm}$ respectively. The PTFE ring mould wrapped with composites is placed on the bottom of the cavity of the glass rotating center. Physical and thermochemical parameters of glass fiber/epoxy composite components and PTFE mould as shown in table 1 . The reaction order is 0.916 and reaction activation energy is $5.46 \times 104 \mathrm{~J} / \mathrm{mol}$.

In the microwave curing oven, the inner wall of the cavity and the waveguide are made of metal. These inner walls can be regarded as perfect electric conductors, so the tangential component of the electric field is zero. The initial temperature of the ring is set at $20^{\circ} \mathrm{C}$, and the initial curing degree is 0.001. During curing, the microwave directly heats the composite ring to cause it cured gradually, and the PTFE mould merely serves to fix and support the composites. In the microwave curing process, as the temperature of the composite ring gradually increases, part of the heat of the composites is transferred to the PTFE mould in the form of heat conduction.

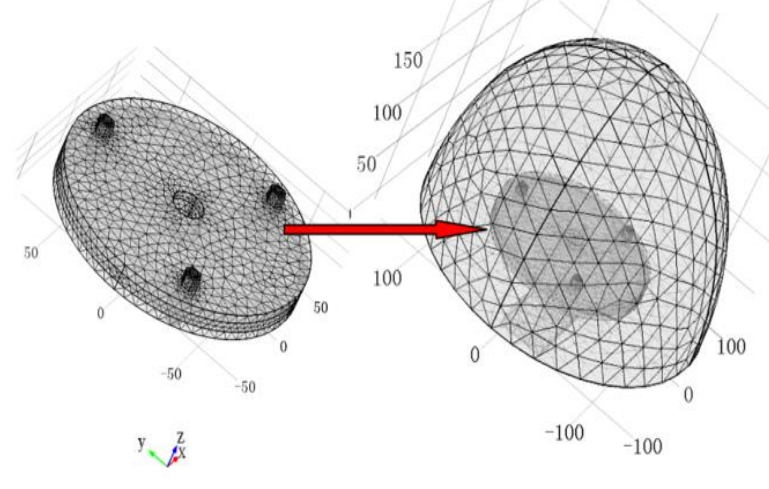

Figure 1. The overall mesh of model.

The finite element method is used to simulate the microwave curing process, and the model is meshed by tetrahedral meshing. Due to the more attention to the distribution of the electric field at the ring of the composites, the mesh at the ring and its mould is refined. The overall meshing situation is shown in Figure 1. A total of 83,286 grid units and about 429,815 degrees of freedom are divided.

In order to simplify the calculation, the basic assumptions made in the numerical simulation are as follows: (1) suppose the resin does not flow during the curing of the composite; (2) assuming that the physical parameters such as density, specific heat and thermal conductivity of the composite are constant; (3) do not take into account the deformation of the composite during curing; (4) in view of the dielectric loss glass turntable and PTFE mould are low, without considering the effect of microwave heating on the two kinds of medium in numerical simulation. 
Table 1. Physical and thermochemical parameters

\begin{tabular}{crcc}
\hline value & \multicolumn{3}{c}{ parameter } \\
\hline & density $\left(\mathrm{kg} / \mathrm{m}^{3}\right)$ & specific heat capacity $\mathrm{J} /(\mathrm{kg} \cdot \mathrm{K})$ & thermal conductivity $\mathrm{W} /(\mathrm{m} \cdot \mathrm{K})$ \\
fiber & $2.54 \times 10^{3}$ & $7.37 \times 10^{2}$ & 1.250 \\
resin & $1.14 \times 10^{3}$ & $1.24 \times 10^{3}$ & 0.165 \\
PTFE & $2.19 \times 10^{3}$ & $1.05 \times 10^{3}$ & 0.244 \\
\hline
\end{tabular}

\section{Results and discussion}

\subsection{Electric field distribution}

The ring mould wrapped with composites is horizontally placed at the center of the microwave curing oven turntable. The height of the lower end side of the ring from the bottom of the cavity is $0.012 \mathrm{~m}$. For this reason, the electric field distribution at the height of $\mathrm{z}=0.012 \mathrm{~m}$ is selected for analysis. Figure 2 and Figure 3 are respectively the electric field distribution cloud at this altitude under no-load and load states.

In the no-load state, the electric field distribution in the microwave curing oven is not uniform enough, but it is worth noting that due to the spherical symmetry of the cavity shape, the electric field distribution also shows obvious symmetry characteristics. Observations also found that the electric field intensity in the vicinity of the waveguide was significantly higher than that in other regions, and due to the spherical reflection effect of the cavity, another approximately symmetrical strong electric field region appeared at the center of the cavity bottom.

When the composite ring is placed at the bottom of the cavity, the electric field distribution in the cavity changes obviously compared with no-load, which indicates that the addition of lossy medium will have a great influence on the electric field distribution. It is considered that that the addition of ring enhances the absorption and reflection of the microwaves, so that the number of electromagnetic field modes inside the cavity of the oven increases, and the electric field is redistributed. From Fig. 3 shows that the electric field intensity inside the cavity under load is significantly higher than that under no-load conditions, and the electric field intensity on the ring is higher than the electric field strength in the surrounding area, mainly due to the electric field distribution has a characteristic to concentrate on areas with lossy media.

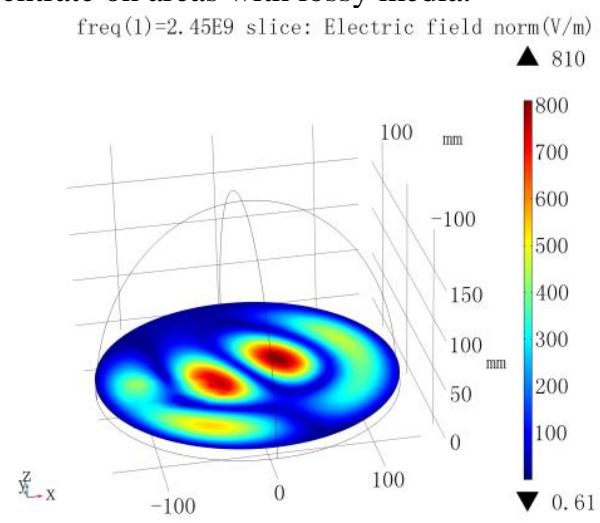

Figure 2. Electric field distribution cloud chart under no-load $\operatorname{state}(\mathrm{z}=0.012 \mathrm{~m})$

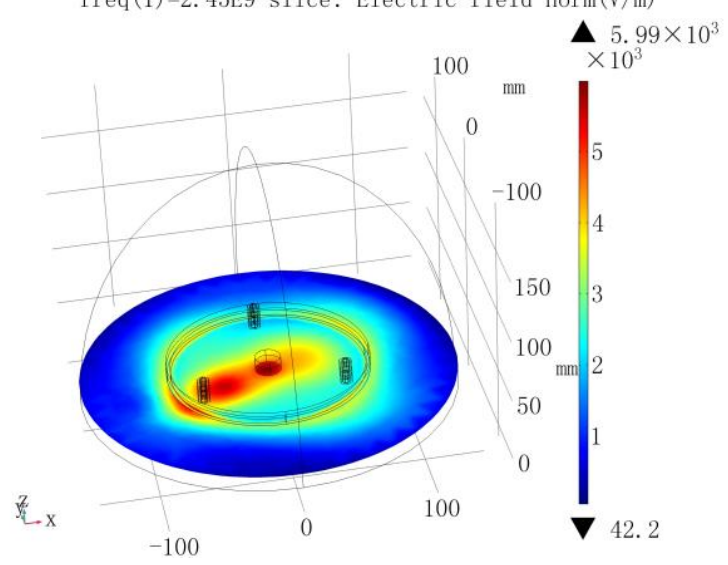

Figure 3. Electric field distribution cloud chart under load state $(\mathrm{z}=0.012 \mathrm{~m})$

The distribution of electric field on the composite ring is an important factor affecting the curing effect of microwave.The more uniform the electric field distribution is, the more uniform the composites are heated during the microwave curing process, and the smaller the gradient of the curing degree is.Fig. 4 shows the cloud distribution of the electric field on the composite ring. It can be seen from the figure that the electric field distribution on the composite ring has some inhomogeneity. The results show that the strength of the electric field in the ring is mainly concentrated in the range of $3000-4000 \mathrm{~V} / \mathrm{m}$, but in the local area close to the waveguide, the electric field intensity of the composite ring is slightly higher and the maximum electric field strength can reach $5827 \mathrm{~V} / \mathrm{m}$. In the local edge region, the electric field strength of the composite ring is slightly lower, and the minimum electric field strength value is $1751 \mathrm{~V} / \mathrm{m}$.

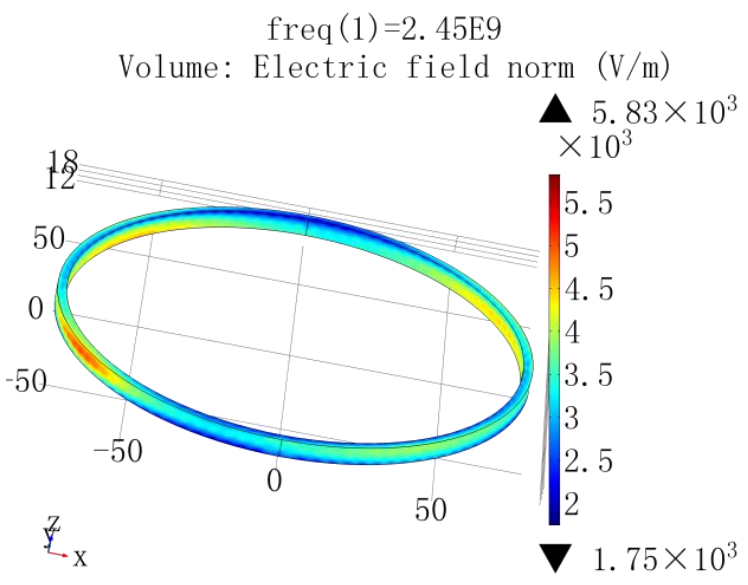

Figure 4. The cloud distribution of the electric field. 


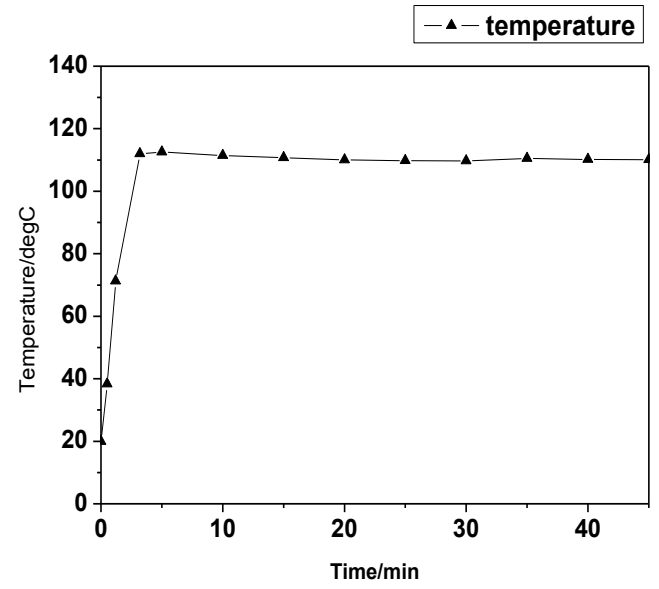

Figure 5. The curve of the temperature at the monitoring point with time.

\section{2. temperature analysis}

A point on the center line of the outer surface of the composite ring is arbitrarily selected as the temperature monitoring point, and the curve of the temperature at the monitoring point with time is shown in Figure 5. It can be seen from Figure 5 that under the microwave irradiation, the temperature of the composites increases rapidly, and the temperature at the monitoring point reaches the curing temperature $110^{\circ} \mathrm{C}$ at 3.2 minutes. After $3.2 \mathrm{~min}$, the monitoring point temperature is controlled at about $110^{\circ} \mathrm{C}$.

Figure 6 shows the temperature distribution of the composite ring at different time. At the initial stage, as the temperature of the composite ring gradually increases, the temperature difference between the inner and outer surfaces gradually increases. When heated to $3.2 \mathrm{~min}$, the monitoring point temperature reaches $110^{\circ} \mathrm{C}$, at this time, the temperature difference between the inner and outer surface of the ring is about $5^{\circ} \mathrm{C}$. It can be analyzed that the difference of the electric field distribution inside the composite ring is the main factor leading to the uneven temperature distribution. The electric field intensity near the inner edge of the ring is obviously higher than that in other regions, which makes the temperature rise in the region a little faster than that in the other regions, and the temperature is not controlled in the heating stage, which makes the temperature of the high electric field on the ring accumulates continuously, thus increasing the inhomogeneity of the temperature distribution. In addition, during the microwave curing process, the natural convection heat transfer between the outer surface and the air of the composite ring can also cause the loss of heat on the outer surface of the ring, which would increase the difference of the temperature distribution between the inner and outer surface of the ring.

When heated to $25 \mathrm{~min}$, the temperature difference between the inner and outer surfaces of the ring is only within $1{ }^{\circ} \mathrm{C}$, indicating that the temperature inside the composites has basically reached the same. It can be analyzed that the control of temperature makes the heat transfer from the high temperature part to the low temperature part and improves the uniformity of the temperature distribution in the composites. Secondly, with the progress of the curing process, the temperature of the air in the oven chamber increases continuously, which weakens the convective heat transfer between the outer surface of the ring and the air to a certain extent, thus reducing the heat loss on the outer surface of the ring.

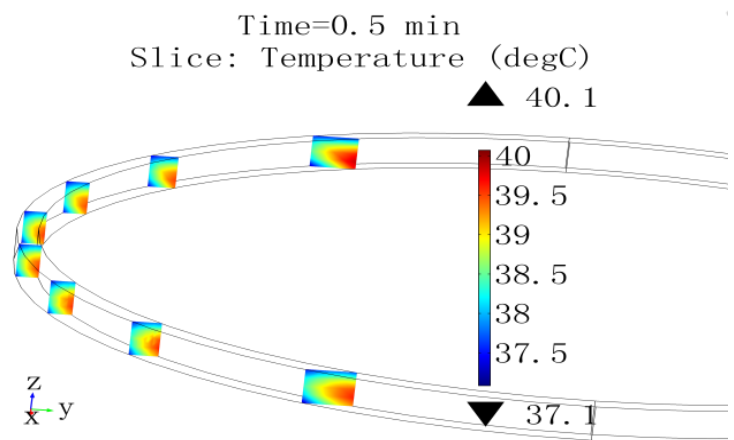

Time=3. $2 \mathrm{~min}$

S1ice: Temperature (degC)

- 115

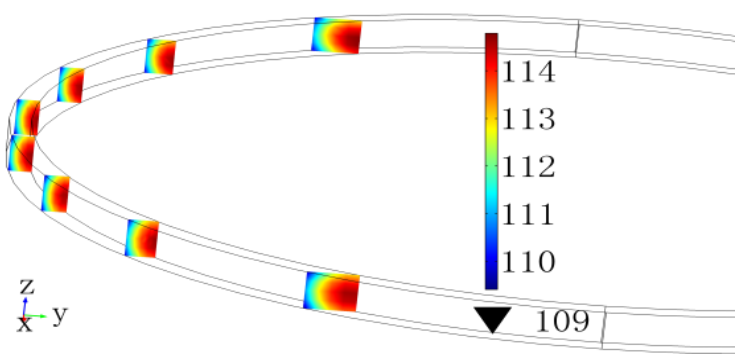

$$
\begin{gathered}
\text { Time }=10 \text { min } \\
\text { S1ice: Temperature (degC) }
\end{gathered}
$$
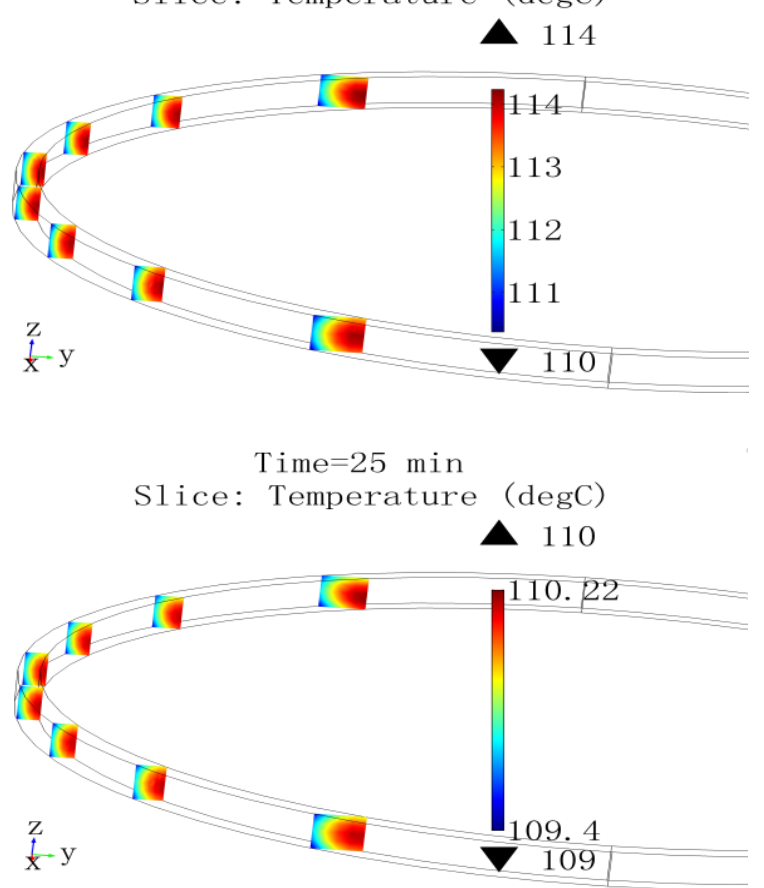

Figure 6. Temperature distribution of composite ring at different time.(YZ - plane slice)

\section{Conclusions}


Based on the theories of electromagnetics, heat transfer and solidification kinetics, a coupled model of electromagnetic field, temperature field, curing degree field for microwave curing process of glass fiber/epoxy composites with two internal heat sources is established. It can be found that the inhomogeneous electric field distribution leads to the difference of temperature distribution inside the composite ring during the initial stage of microwave curing, and the convection heat transfer between the outside of the ring and the air also increases the temperature difference. As the curing process carries on, the temperature difference will gradually decrease due to the control of the curing temperature. Therefore, it is necessary to take appropriate temperature control measures in the initial stage of microwave curing, in order to reduce the adverse effect of the difference of electric field intensity distribution on the heating rate and temperature distribution of the composites.

\section{References}

1. Li Y G, Li N Y and Gao J 2014 J. International Journal of Advanced Manufacturing Technology. 70 591-606.

2. Li S J, Zhan L H, Chen R, et al 2014 J. Fibers and Polymers. 152 404-09.

3. Joshi S C and Bhudolia S K 2014 J. Journal of Composite Materials. 48 3035-48.

4. Hassan M R and Ganjeh B 2014 J. Applied Mechanics and Materials. 564 310-14.

5. Durka M K, Dimitrakis G and Bogdal D $2015 \mathrm{~J}$. Chemical Engineering Journal. 264 633-44.

6. Nesbitt A, Navabpour P, Degamber B, et al $2004 \mathrm{~J}$. Measurement Science and Technology. 15 2313-2324.

7. Ma R L, Chang X L, Liao Y Q and Zhang X J 2016 J. Polymer Material Science and Engineering. 32 96-101.

8. Ma R L, Chang X L, Liao Y Q and Zhang X J 2016 J. Journal of Solid Rocket Technology. 39 809-14. 